\title{
THE RETICULOCYTE CRISIS AFTER THE EFFECTIVE SPECIFIC TREATMENT OF PERNICIOUS ANAEMIA
}

\author{
BY \\ RAGNAR BERLIN \\ From the Medical Clinic, the University Hospital, Uppsala, Sweden
}

(RECEIVED FOR PUBLICATION SEPTEMBER 25, 1950)

Since liver extracts began to be used in the treatment of pernicious anaemia animated discussion has been carried on with regard to the cause of the reticulocyte crisis that then occurs. The phenomenon is by no means easy to explain, and at first sight it would seem absurd that following effective treatment of pernicious anaemia only a sudden, transient increase in the reticulocytes can be obtained, despite the fact that the red blood cells subsequently continue to rise to normal values.

The simplest way of getting over the difficulty is to assume, as many investigators have already done, that the remission in pernicious anaemia takes place without the newly formed red blood cells passing through the normal reticulocyte stage. No really conclusive proof of the correctness of this assumption has as yet been advanced.

After Singer, King, and Robin (1948) had reported their discovery that blood corpuscles transfused from untreated cases of pernicious anaemia to normal children had a much shorter survival time (28-75 days), but after treating the patients with a liver extract their newly formed red blood cells had a normal life cycle, the discussion was transferred to some extent to other aspects of the problem. It was then thought that the initial increase in the reticulocytes was due to enormous hyperactivity of the bone marrow following the treatment, and that a number of more or less defective blood cells were produced before and during the early remission and then rapidly eliminated in the peripheral circulation. After the production of red cells has begun to function effectively, however, more and more normal erythrocytes with a normal lffe cycle were formed and consequently there was no longer any need for such an intensive delivery of newly formed blood cells per time unit. This was the explanation advanced to account for the decrease in the reticulocytes. It is obvious, however, that this explanation is not satisfactory when it is subjected to a critical analysis. In pernicious anaemia it is the rule that no pronounced increase in the number of red blood corpuscles is observed in the reticulocyte crisis. It is only after some time has passed that the blood values begin to show a more or less steady tendency to rise, and the need of full activity in the blood-cell factories therefore seems undeniable. The more stable structure of the newly formed blood corpuscles, which would cause among other things a normal life span in vivo, could hardly be expected to make itself felt before-at the very 
earliest-the time when the previously formed, defective blood corpuscles had completed their life cycle ; in other words, according to the investigations of Singer et al., until after 28 days. I have repeated Singer's experiment and was able to confirm his statements to the full; in one case, however, the life span of the megalocytes showed the relatively low figure of 18 days. Consequently, the change in the length of the life cycle definitely cannot explain the abrupt drop in the reticulocyte count. If blood from a pernicious anaemia patient during the upward swing of the reticulocyte crisis is transfused to a normal person (Berlin, 1950), the maturation curve for the reticulocytes in vivo, which is obtained by determining the reticulocyte value in the recipient's blood immediately after the transfusion and then at frequent intervals during the subsequent 24 hours, will be as shown (Fig. 1a). If the blood is transfused just at the time when the reticulocyte peak is reached another type of curve is obtained (Fig. $1 b$ ), and if the blood is taken after the crisis is over and the reticulocytes are on the decrease again the maturation curve will have still another appearance (Fig. 1c). It will be seen that the total maturation time, roughly $17-18$ hours, is surprisingly similar in all three instances. The course of the curve in Fig. $1 a$ may be explained by the fact that the distribution of cells of different ages among the transfused reticulocyte population was uneven, and that older, more mature cell-types were in the majority. After a short time the older forms developed rapidly after entering a normal environment, and the final, more horizontal part of the curve in all probability shows the maturation of the youngest, newly liberated reticulocyte forms which later on developed in the recipient's circulation within the normal time, i.e., 18 hours. Fig. $1 b$, on
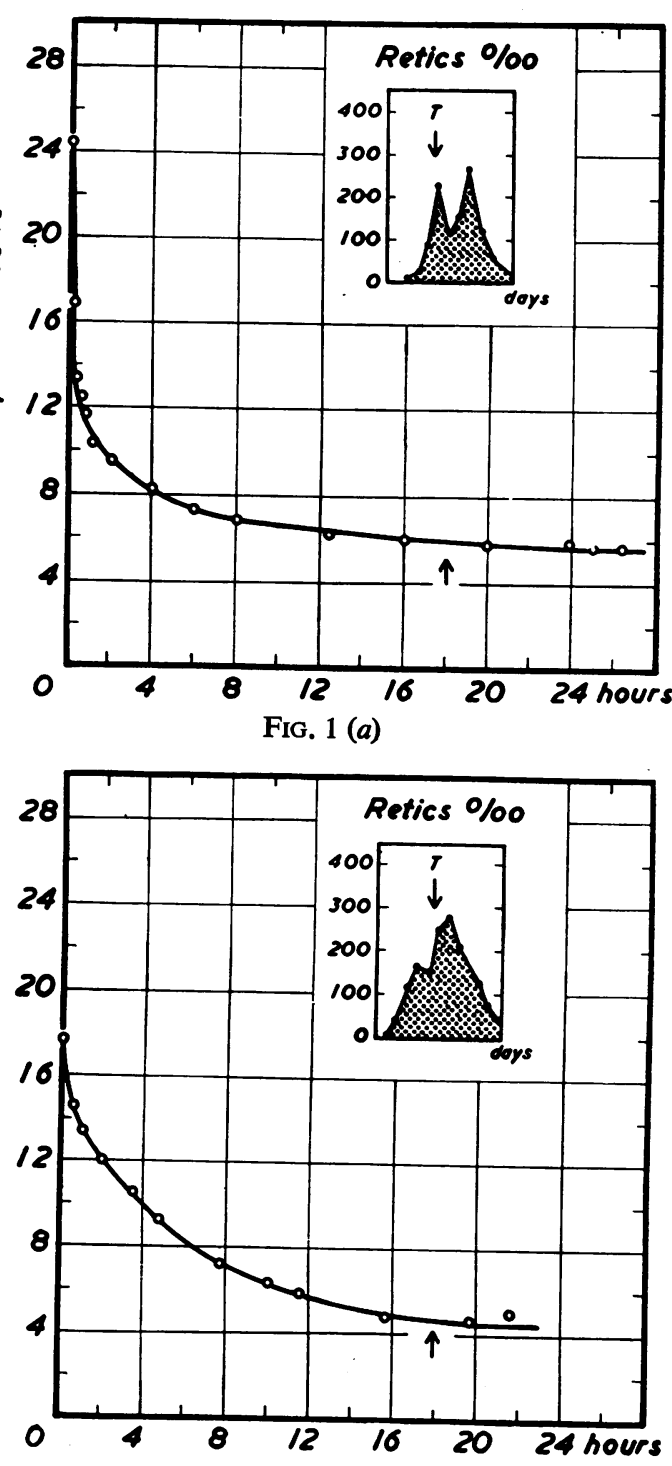

FIG. 1 (b)

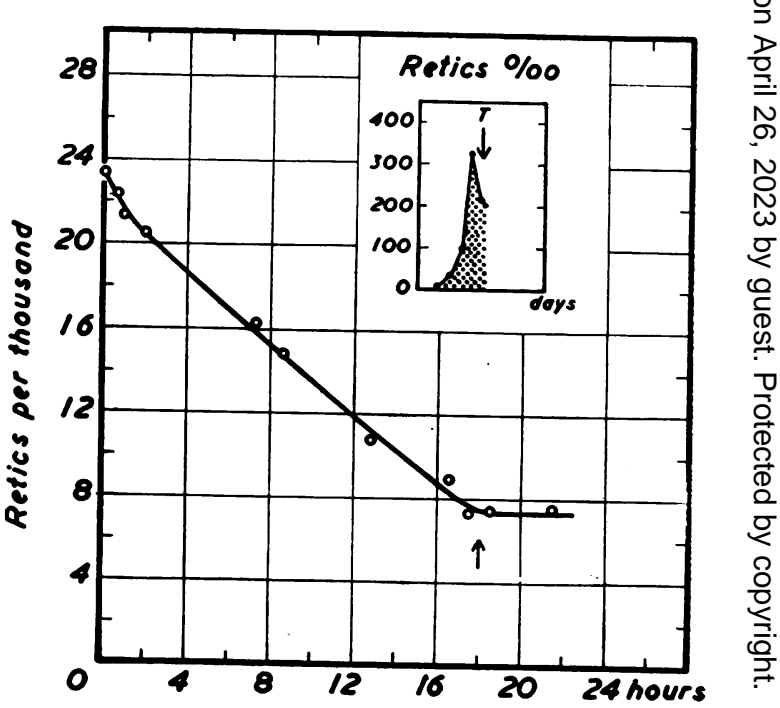

Fig. $1(c)$ 
the other hand, with a more even slope probably indicates that the different ages of the reticulocytes were more evenly distributed. The third type of curve (Fig. 1c) would seem to indicate that there was a preponderance of young, newly produced forms among the transfused reticulocytes. To obtain accurate information 10,000 cells have been counted on every slide. The standard deviation in these calculations has not exceeded $8 \%$ of the mean. On the basis of these experimental observations the supposition might be advanced that the maturation time of reticulocytes at the start of the reticulocyte peak is considerably prolonged; at that stage relatively old, more highly developed reticulocytes are over-represented-in patients with pernicious anaemia - but the maturation process rapidly begins to function in the recipient's normal environment. After the reticulocyte peak the conditions would be the reverse, the maturation of the reticulocytes now proceeds more rapidly, and consequently a sudden drop in the number of reticulocytes is found, and the cells consist, for the most part, of newly formed immature forms.

It is surprising how little attention has been paid to the investigations of Jörgensen and Plum (1943) and Ruth Plum (1947) into the maturation of reticulocytes. These authors have demonstrated that there is a special reticulocyte ripening factor in the blood, and they have made a study of this factor under normal and pathological conditions. They point out that in untreated pernicious anaemia patients there is a considerably lowered amount of the active ripening factor in plasma, but during effective treatment this increases not only to normal values but even to values exceeding the normal figures. They have also shown that considerable amounts of the ripening factor can be found in pernicious anaemia patients but that it occurs in an inactive form as long as the disease remains untreated. These observations throw further light on the interpretation of the in vivo reticulocyte-maturation curves and strengthen the assumption that at the beginning of the reticulocyte crisis there is increased erythropoiesis, set in motion by the liver therapy, but that the increase, or activation, of the reticulocyte-ripening factor, which should follow the treatment, has not yet come fully into action. The result will then be that the newly formed blood corpuscles remain at the reticulocyte stage for a longer time than normally, perhaps for several days. Consequently, there must be a considerable increase in the number of reticulocytes in the peripheral blood, and the majority will be relatively old cells. When the curve has reached its peak the amount of substance able to transform reticulocytes into mature red cells has, however, increased in the blood, the previously older cells develop rapidly into non-reticulated cells, and the newly formed reticulocytes also quickly develop. There is then a steep drop in the curve, which corresponds to a decrease in the reticulocytes. During the entire remission the maturation factor is present in normal or more than normal amounts, and, despite the fact that erythropoiesis is still very lively, the reticulocytes do not increase very much above the normal figure. It is thus unnecessary to have recourse to the unverified statement that the cells produced as the remission proceeds are liberated from the bone marrow without passing through the reticulocyte stage. This reasoning also receives support from the well-known observation, that, if a pernicious anaemia patient is given a suboptimal dose of liver extract so that a pronounced reticulocyte response is obtained but no real remission starts, 
the blood values remain constant, the serum iron level tends to rise, and the sternal marrow begins, in some cases, to display megaloblast proliferation: when effective treatment is instituted later the reticulocyte response is extremely slight. In accordance with this line of reasoning, there would then still be enough maturation factor left, and consequently the reticulocyte crisis could not develop. These results would explain the findings of Davidson, Murphy, Watson, and Castle (1946), who found that a patient with pernicious anaemia, transfused to bring the blood up to normal levels, failed to produce a reticulocyte crisis when given liver extract. This has previously been interpreted as being due to the fact that, by bringing the patient's blood up to nearly normal values, the new formation of red blood cells was hindered. It cannot be denied, however, that this result might just as well be a consequence of the fact that with the transfused blood such large amounts of reticulocyte-ripening factor had been supplied that it had sufficed to prevent the occurrence of a reticulocyte crisis in response to the subsequent liver therapy.

\section{Summary}

The current views regarding the mode of origin of the reticulocyte crisis following liver treatment in pernicious anaemia is analysed. Experiments are described which show that, if blood from pernicious anaemia patients is transfused during the reticulocyte crisis to normal persons under different conditions, the curves of the reticulocyte development that are obtained by determining the number of reticulocytes in the recipient's blood at frequent intervals after the transfusion will also be quite different. It is considered probable that when blood cells are transfused at the beginning of the reticulocyte crisis there will be a rapid initial development of a high percentage of older reticulocytes. If, on the other hand, the blood is transfused when the reticulocyte peak is on the wane, the drop noted in the maturation curve suggests that in this instance the population is chiefly of young, immature cells.

These observations are compared with the investigations into the reticulocyteripening factor occurring in pernicious anaemia reported by Jörgensen and Plum and by Ruth Plum ; these authors found a reduced amount of active factor in untreated cases of pernicious anaemia. The amount of this substance in plasma rises to values almost higher than normal during the reticulocyte crisis.

It is thought that the change in the development rate of the reticulocytes at the beginning and at the end of the reticulocyte crisis in all probability is the explanation of this otherwise incomprehensible phenomenon.

\section{REFERENCES}

Berlin, R. (1950). Scand. J. clin. Lab. Invest., 2, 37.

Davidson, C. S., Murphy, J. C., Watson, R. J., and Castle, W. B. (1946). J. clin. Invest., $25,858$.

Jörgensen, S., and Plum, C. M. (1943). Ugeskr. Laeg., 105, 787.

Plum, R. (1947). Reticulocytmodningsindex under normale og patologiske forhold. Thesis, Copenhagen.

Singer, K., King, J. C., and Robin, S. (1948). J. Lab. clin. Med., 33, 1068. 